\title{
Designing and Evaluation of Performance of a 256- Heirarchical QAM for Image Transmission
}

\author{
Sarabjit Kaur \\ Department of ECE \\ I.P. University, Delhi, India
}

\begin{abstract}
Transmission of multimedia, especially transmission of image/video information, requires high reliability and high digital transmission speed. But, in case of mobile communication systems, since transmission reliability is degraded by multipath fading also the frequency bandwidth is severely limited, it is difficult to realize the multimedia transmission without applying techniques to overcome these limitations. For achieving a high quality and high speed in digital image transmission in a band-limited fading channel, Hierarchical modulation is a better method. This method is also constellation is used to give different degrees of error protection for the most important information of the image content. A higher order i.e. 256-HQAM where non-uniform signal constellation with hierarchical oriented OFDM modulator has been proposed. Proposed method is used to provide lesser BER and Bandwidth efficiency in the compressed image data at lower channel Signal to Noise Ratio (SNR). An information theoretical approach is applied to analyze the performance of proposed technique. Using the approach, it is quantitatively evaluated how much the proposed technique outperforms. BER and PSNR of reconstructed image of proposed methodology, which include uniform and non-uniform constellations, over AWGN environments, have been calculated to analyze the performance. The performance of proposed and existing technique has been evaluated by doing simulation using MATLAB 7.6 (R2008a).
\end{abstract}

\section{KEYWORDS}

LP, HP, Modulation, HQAM, Constellation.

\section{INTRODUCTION}

There are two major concerns, bandwidth limitation and high probability of error for transmission of images over wireless communication channels. Therefore, compression is applied to the transmitted data in order to conserve the bandwidth. However, compression increases bit dependency that in turn introduces error extension effects. Image compression standards cannot be directly transferred to a hostile mobile environment as they have been developed for relatively error free environments. It is due to the extensive employment of variable length coding techniques which are very much error sensitive, because a single transmission error may result in a uudecoded string of bits. Therefore a problem arises in transmission of highly error sensitive image information against hostile mobile environments [5], [6]. There are several error resilient image coding techniques [4] which are designed to minimize the effects of the transmission errors on the reconstructed image quality. Unequal error protection (UEP) of coded image bit-stream is one of the most successful techniques. Unequal error protection (UEP) uses the idea which is based on the fact that bits in a compressed image stream have unequal importance. For example the motion vectors and picture header are much more important than the texture image data. The reconstructed image quality will be severely degraded when errors occur on the important bit stream. So, as to do so, important bits should be given a higher protection and priority other than the rest of the image bit-stream. There is one more serious problem, which is very unique to wireless networks is the extremely hostile and random nature of the channels. This nature of channels introduces distortion and considerably degrades the image quality. Error control coding is used to control the errors, however, the addition of check bits that carry no information further increases the data length and consequently the bandwidth [1]. This paper proposes a 256 HQAM arrangement, with hierarchical oriented OFDM modulator for the digital image transmission, which does not use the concept of unequal error protection.

It is a modification of Quadrature Amplitude Modulation (QAM) and conventional HQAM to accomplish the same objectives. One major drawback of conventional HQAM is that there are fixed allocated capacities for the high priority (HP) and low priority (LP) data layers. But, this is a simple and efficient approach in which non-uniform signalconstellation has been used to give different degrees of protection to the transmitted bits. The main advantageous feature of this method is that different degrees of protection are achieved without an increase in bandwidth in contrast to channel coding that increases the data rate by adding redundancy to the transmitted signal [2]. HQAM provides better degree of protection [8]. HQAM provides better transmission through noisy channels Increase in value of modulation parameter results in low BER and increase in PSNR. HQAM is extended form of QAM. HQAM provides more coverage area for important information.

BER analysis has been presented for different values of the modulation parameter i.e. alpha and performance comparison is carried out through MATLAB simulation using 256HQAM with gray image as test image.

\section{HIERARCHICAL QAM}

Hierarchical modulations were initially proposed to provide different classes of data to users in different wireless reception conditions [3]. The 16-QAM constellation naturally forms two different-integrity sub-channels. Fig.1 show the constellation of 16-HQAM modulation, where $\mathrm{b}$ and $\mathrm{c}$ are the minimum distance between points inside each quarter. 


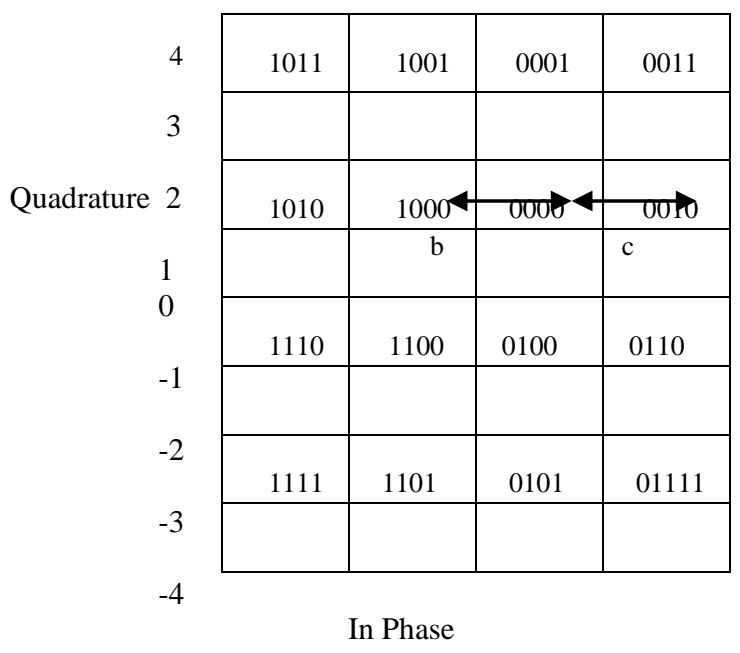

Fig. 1: constellation of 16-HQAM modulation

The first sub-channel (HP) is formed by the two most significant bits (MSBs) of the four bit symbol, and the second sub-channel (LP) is formed by the two least significant bits (LSBs) of the symbol. Bits transmitted via the HP sub-channel are received with a lower probability of error than those transmitted via the LP sub-channel [3]. The splitting of the symbol into sub-channels leads to improved Bit Error Rates (BER) for the channels that carry the most important information of the video. The bits from the H.264 video source encoder that are most sensitive in terms of picture quality are assigned in the HP sub-channel and the remaining bits assigned in the LP sub-channel.

To improve the transmission efficiency of the system, higher error protection can be applied to the most important data of the coded video data by using 16-HQAM with $\alpha>1$, where alpha is the ratio of between the minimum distance between quadrants (b) and minimum distance between points inside each quadrant (c) and so is given by

$$
\alpha=b / c
$$

In this case the performance of the HP will be improved at the expense of LP.HQAM helps in fulfilling all essential requirements of broadcasters [9]. This is a simple and efficient approach in which non-uniform signal-constellation is used to give different degrees of protection to the transmitted bits. The advantage of this method is that different degrees of protection are achieved without an increase in bandwidth in contrast to channel coding that increases the data rate by adding redundancy to the transmitted signal [2, 10-12]. More programs can be accommodated due to low data rate of LP. HQAM can be used in various multimedia applications. Since mobile services require higher field strength than for fixed services, so good coverage could be achieved to fixed receivers of the LP stream.

\section{SIMULATION OF HIERARCHICAL MODULATION}

The simulation of Hierarchical modulation is based on adjustment of existing QAM modulation algorithm from Communication Toolbox. Function modem.qammod (M) creates M-QAM modulator object, that can be used with function modulate from Signal Processing Toolbox. Property SymbolOrder of the modulator object determines order in which symbols will be mapped. The default value is 'binary' so it could be changed to 'gray'. This can be solved by setting symbol order property to 'user-defined'. This allows change the modulator property SymbolMapping, in which decimal symbol values are stored, when read column wise, starting in the left upper corner of the constellation diagram. For example, to properly map 16-QAM constellation, it has to be defined: Symbol-Mapping $=\left[\begin{array}{l}8 \\ 9\end{array} 13121011151423760\right.$ $154]$.

Steps described above provide us properly mapped symbols into uniform QAM constellations. To map nonuniform constellations, complex values of mapped symbols have to be changed to spread the constellation quadrants apart. Values of $I$ and $Q$ parts of the complex symbols are stored in modulator property Constellation, however this property is read-only and cannot be unlocked for user values like in case of Symbol Mapping property. A simple yet fully functional solution was implemented. First, symbols are mapped into uniform constellations as described above. Then, constellation points are moved on the real axis, depending on the constellation ratio or modulation factor $\alpha$

$$
\begin{aligned}
& Z n=Z n+(\alpha-1) \operatorname{Re}\{Z n\}>0 \\
& Z n=Z n-(\alpha-1) \operatorname{Re}\{z n\}<0
\end{aligned}
$$

next, constellation points are moved on the imaginary axis using the same rule.

$$
\begin{aligned}
& \mathrm{Zn}=\mathrm{Zn}+\mathrm{i}(\alpha-1) \operatorname{Im}\{\mathrm{Zn}\}>0 \\
& \mathrm{Zn}=\mathrm{Zn}-\mathrm{i}(\alpha-1) \operatorname{Im}\{\mathrm{Zn}\}<0
\end{aligned}
$$

The obtained signal is ready for modulation on the RF carrier, using quadrature modulator [7], and its transmission through the channel can be simulated. The carrier is demodulated and channel correction is performed as the first step on the receiver side. Since the pilot subcarriers, used for channel estimation, are generated by the same process as in case of uniform constellations.

Next processing steps are exactly opposite to the modulator. The signal is divided by the scaling factor and opposite transformation to the (2) and (3) is applied to receive uniform, properly scaled constellation, which can be demodulated using standard Matlab demodulator. Demodulator object modem.qamdemod (M) is created using the same mapping settings as the previously described modulator and demodulation is performed by demodulate function.

\section{PROPOSED METHODOLOGY FOR 256-HQAM}

- $\quad$ Reading of RGB image and getting 3D equivalent image matrix.

- Computing approximation coefficients and details coefficients matrices through Discrete Wavelet Transform.

- Getting wavelet coefficients by Wavelet decomposition of the approximation coefficients matrix at level N.

- Setting of a suitable threshold value.

- De-noising and compression of wavelet coefficients and thresohlding using global positive threshold value.

- Single-level wavelet reconstruction of approximation coefficients matrix, based on approximation matrix and (horizontal, vertical, and diagonal) details matrices $\mathrm{CH}$, $\mathrm{CV}$ and $\mathrm{CD}$.

- $\quad$ Getting of reconstructed compressed image. 
- Conversion of components of compressed image into binary equivalent.

- Conversion of binary values into gray coded equivalent values.

- Creation of Inphase and Quadrature matrix so as to create a 256 QAM constellation matrix.

- Real part of constellation matrix is consisted of elements from In-phase matrix.

- Complex part of constellation matrix is consisted of elements from Quadrature matrix.

- $\quad$ Selection of input SNR.

- Selection of modulating factor (alpha) which is real hierarchy in HQAM.

- Modulation of gray coded input with constellation and generation of new modulated signal.

- $\quad$ Addition of modulating factor from real part which is greater than zero in modulated signals.

- $\quad$ Subtraction of modulating factor from real part which is lesser than zero in modulated signals.

- Addition of modulating factor from complex part which is greater than zero in modulated signal.

- Subtraction of modulating factor from complex part which is lesser than zero in modulated signals.

- $\quad$ Adding of AWGN with hierarchical modulated signal.

- Performing Hierarchical scaling on demodulated signal.

- Demodulation of AWG noisy signal.

- Denoising of noisy signal using wavelet.

- Counting of errors by subtracting the matrices of transmitted and received image.

- Calculation of Bit Error Rate and PSNR for AWGN and RICIAN channel.

- Evaluation of performance by plotting BER vs. alpha

- Plotting of constellation mapped signal.

\section{RESULTS}

A simulation for gray scale image transmission and reception is carried out using 256-QAM and the proposed technique. Results from proposed technique is then compared with those obtained with 256-QAM. All simulations are performed using MATLAB 7.6 (R2008a). First, a simulation has been performed to transmit an image with 256 QAM modulation techniques and then, another simulation is performed for proposed technique. BER for both schemes is calculated with SNRequals to $2 \mathrm{db}$. A plot has been made between BER and different values of alpha. Figure2 shows the original transmitted image. Figure 3 shows reconstructed compressed image using DWT. Figure 4 to figure 7 shows received image at different values of alpha. Figure 8 compares the BER performance of 256QAM and proposed technique, which shows that BER for proposed technique at alpha $=4$, is much lesser than that of 256-QAM i.e. alpha equals to 1 . Figure 9 is for plotting the PSNR values for different values of alpha at input SNR 2dB. Plot is gradually increasing with increasing values of alpha. Figure 10 shows BER performance with different values of $\alpha$ when Rician channel is used. Figure 11 is for plotting the PSNR values for different values of $\alpha$. Plot is gradually increasing with increasing values of $\alpha$. But values of PSNR are low in case of rician channel as compared to AWGN. Figure 12 shows the mapping of modulated signal under proposed method. Also, table 1 and 2 clearly shows the values of PSNR and BER of extracted image at different values of input SNR and alpha for AWGN and Rician channel respectively. BER of extracted image is much lesser in case of HQAM as compared to that of QAM. BER of extracted image for AWGN channel is much lesser than that of Rician channel.
PSNR of extracted image is much higher in case of HQAM as compared to that of QAM. PSNR of extracted image for AWGN channel is much higher than that of Rician channel.

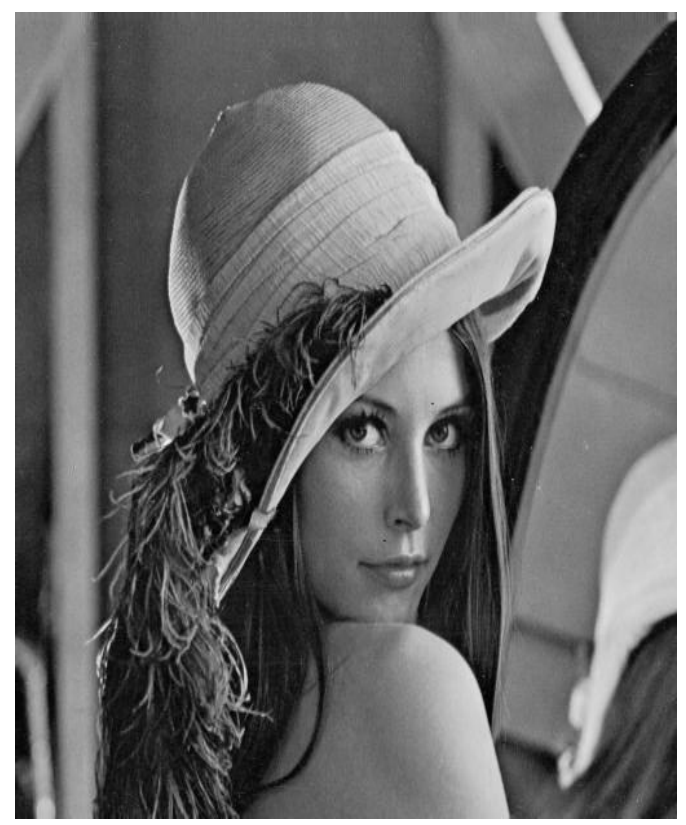

Fig. 2: Transmitted image

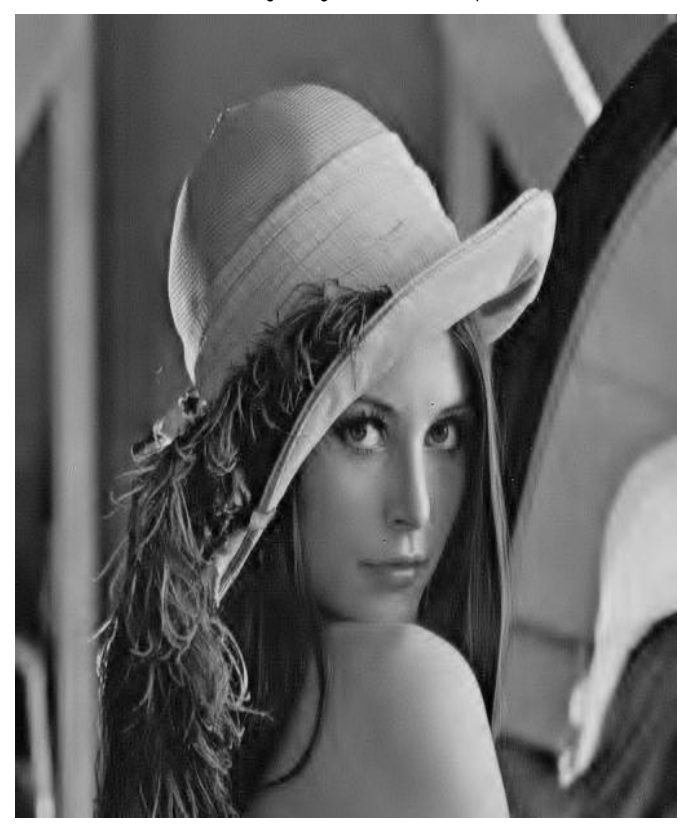

Fig. 3: Compressed image using DWT 


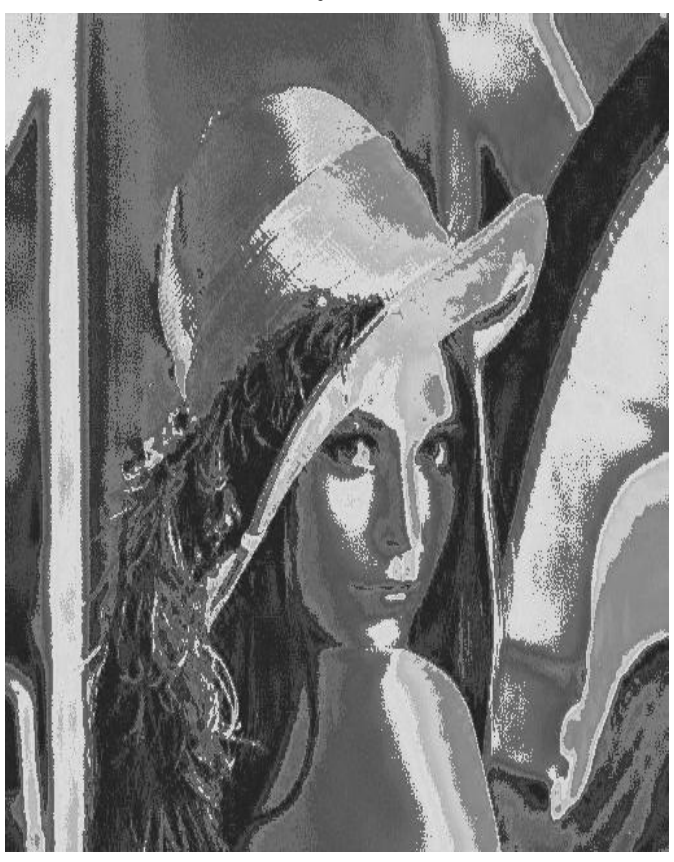

Fig. 4: Received image at alpha=1(with AWGN)

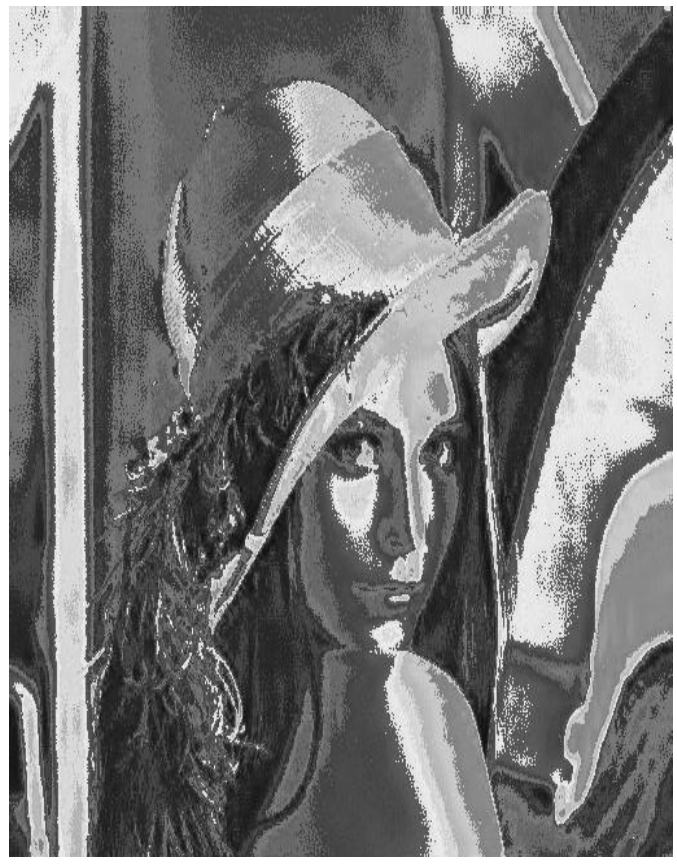

Fig. 5: Received image at alpha=2 (with AWGN)

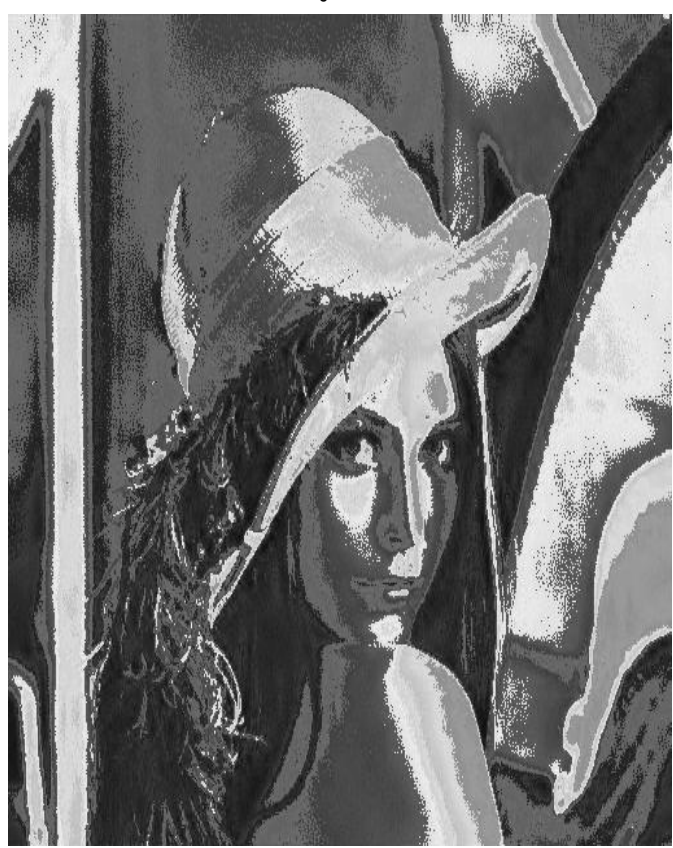

Fig. 6: Received image at alpha=3 (with AWGN)

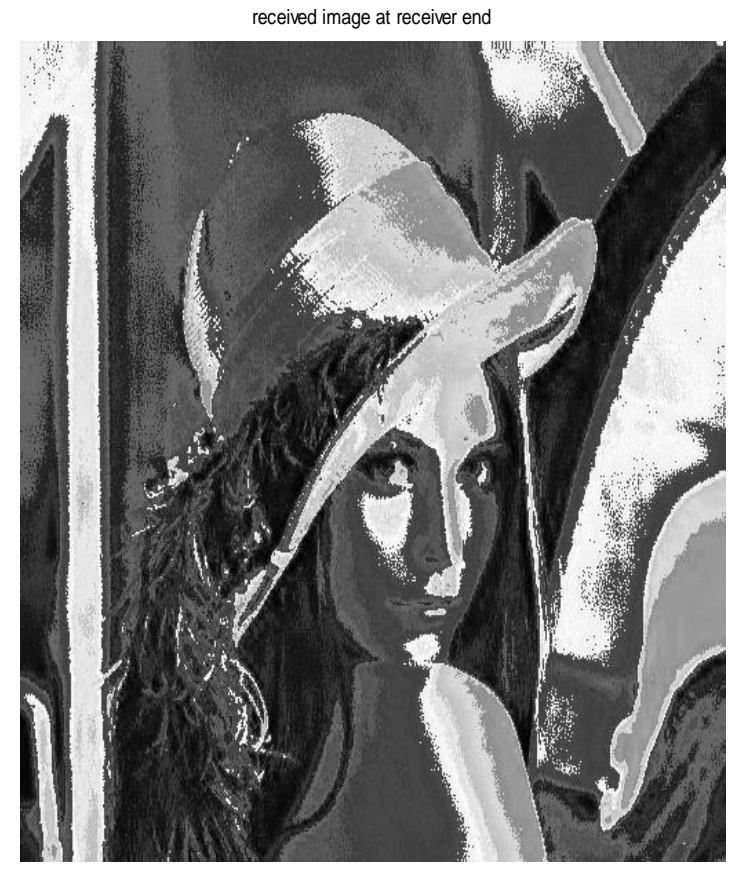

Fig. 7: Received image at alpha=4 (with AWGN) 


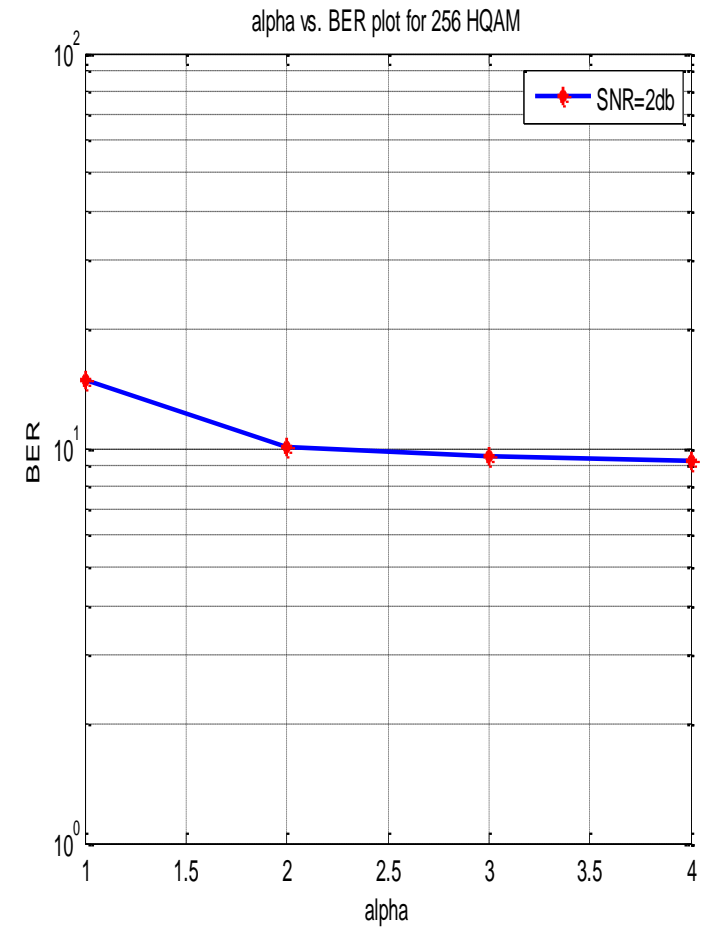

Fig. 8: BER performance with increasing value of alpha (with AWGN)

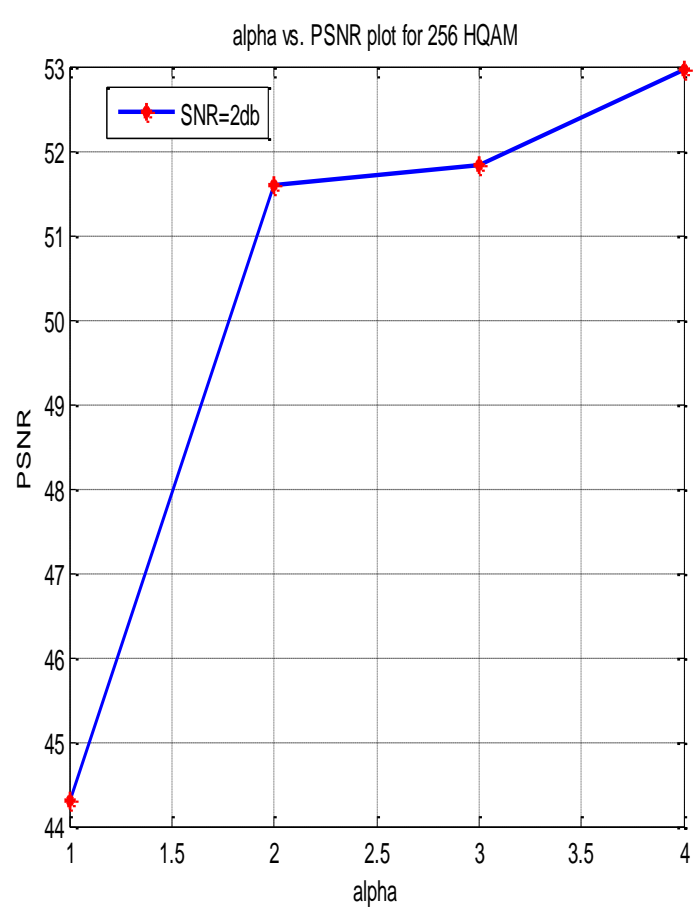

Fig. 9: PSNR vs. alpha (with AWGN)

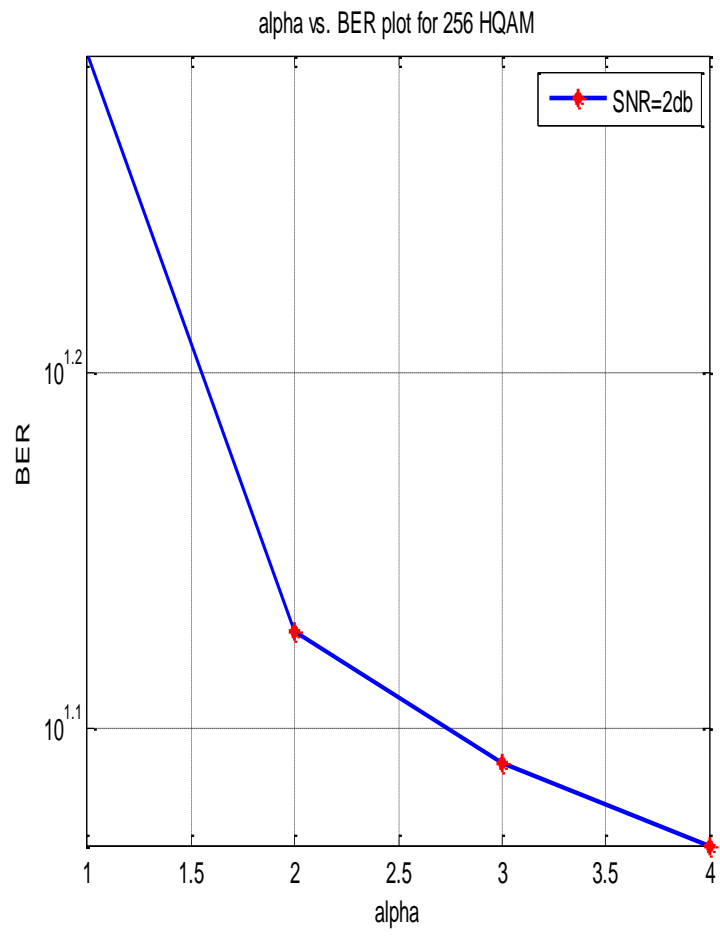

Fig.10: BER performance with increasing value of alpha (with Rician channel)

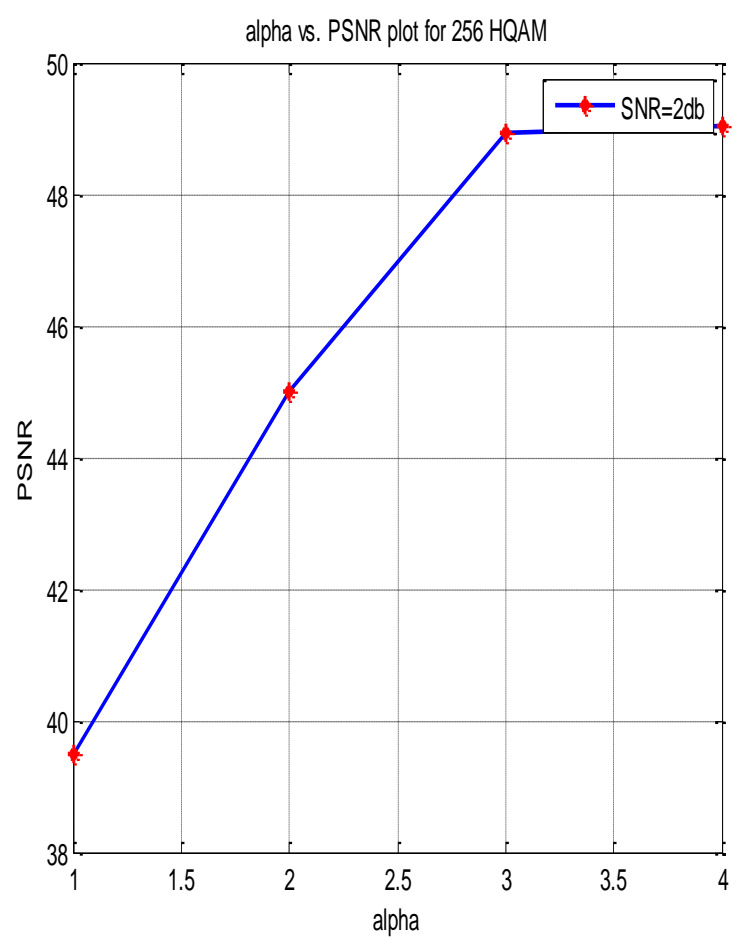

Fig. 11: PSNR vs. alpha (with Rician channel) 


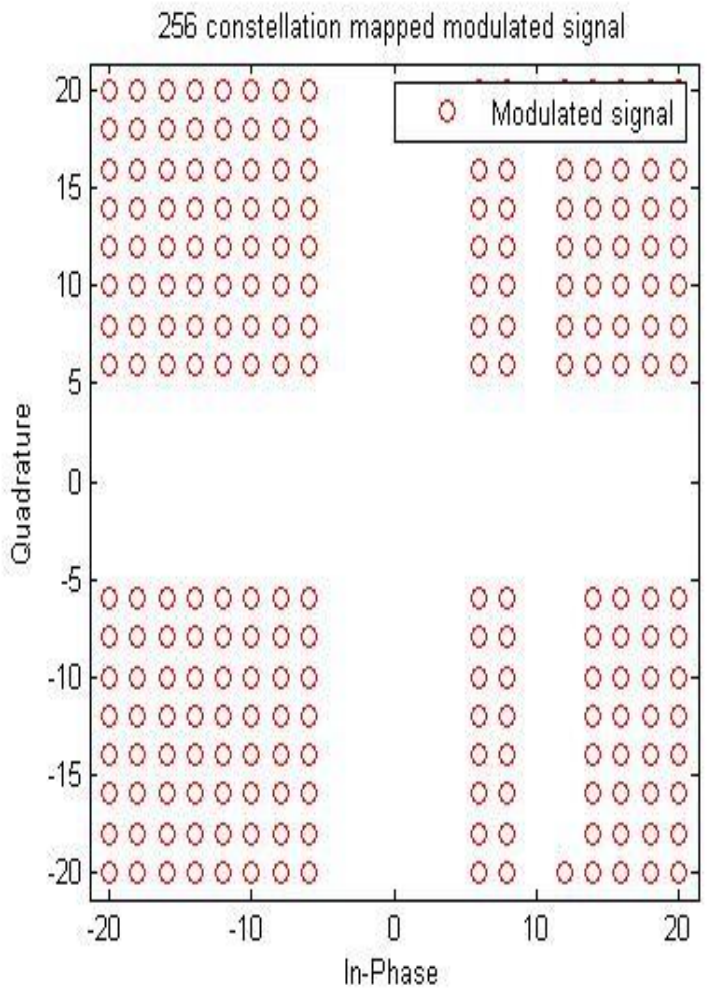

Fig. 12: Constellation mapped modulated signal

Table 1. Comparison of HQAM with QAM using Rician channel at different values of input SNR and alpha for different values of BER and PSNR

\begin{tabular}{|l|l|l|l|l|}
\hline & $\begin{array}{l}\text { At input } \\
\text { SNR=2dB }\end{array}$ & $\begin{array}{l}\text { At input } \\
\text { SNR=3dB }\end{array}$ & $\begin{array}{l}\text { At input } \\
\text { SNR=4dB }\end{array}$ & PSNR \\
\hline alpha=1 & 19.46 & 24.56 & 27.24 & 39.47 \\
\hline alpha=2 & 13.40 & 19.29 & 23.00 & 44.98 \\
\hline alpha=3 & 12.31 & 16.06 & 19.90 & 48.94 \\
\hline alpha=4 & 11.67 & 14.43 & 18.15 & 49.02 \\
\hline $\begin{array}{l}\text { 256- } \\
\text { QAM }\end{array}$ & 24.67 & 33.35 & 41.49 & 46.41 \\
\hline
\end{tabular}

Table 2. Comparison of HQAM with QAM using AWGN channel at different values of input SNR and alpha for different values of BER and PSNR

\begin{tabular}{|l|l|l|l|l|}
\hline $\begin{array}{l}\text { Output } \\
\text { BER for }\end{array}$ & $\begin{array}{l}\text { At input } \\
\text { SNR=2dB }\end{array}$ & $\begin{array}{l}\text { At input } \\
\text { SNR=3dB }\end{array}$ & $\begin{array}{l}\text { At input } \\
\text { SNR=4dB }\end{array}$ & $\begin{array}{l}\text { Output } \\
\text { PSNR }\end{array}$ \\
\hline alpha=1 & 10.06 & 16.24 & 19.23 & 51.60 \\
\hline alpha=2 & 9.52 & 12.24 & 15.98 & 51.84 \\
\hline alpha=3 & 9.28 & 13.12 & 14.37 & 51.95 \\
\hline alpha=4 & 12.9 & 11.75 & 15.45 & 48.78 \\
\hline $\begin{array}{l}\text { 256- } \\
\text { QAM }\end{array}$ & 24.67 & 33.35 & 41.49 & 46.41 \\
\hline
\end{tabular}

\section{CONCLUSION}

A new image transmission over wireless communication system based on the hierarchical transmission techniques has been proposed, which is compared with non-hierarchical
(QAM) transmission technique. In image transmission, QAM provides higher transmission efficiency and low BER by utilizing both amplitude and phase variations. However, it requires higher transmission bandwidth due to its equal protection of high and low priority data bits. Hierarchical QAM (HQAM) overcomes this disadvantage by providing more protection to the higher priority bits and less protection to the lower priority bits of the image data. Also, proposed technique resolves the problem which occurs due to variable bit rate ratio and fixed allocated capacities for the high priority (HP) and low priority (LP) data layers by introducing 256 HQAM arrangement with hierarchical oriented OFDM modulator. This system not only lowers down the BER, but also increases the PSNR of received image. Thus proposed technique being a major modification of QAM technique provides a more efficient means of image transmission over erroneous wireless channel without any additional hardware.

\section{REFERENCES}

[1] Farid Ghani, Md. Abdul Kader, and R. Badlishah Ahmed "Hierarchical Quadrature Amplitude Modulation for Image Transmission Over Erroneous Wireless Channels" International Journal of Video \& Image Processing and Network Security IJVIPNS-IJENS Vol: 11 No: 06.

[2] B.Barmada, M.M.Gandhi, E.V.Jones, M.Ghanbari, "Prioritized transmission of Data Partitioned H.264 Video with HQAM" Signal Processing Letters, IEEE, Vol.12, No.8, pp.577-580, August, 2005.

[3] Ahmed B. Abdurrhman and Michael E. Woodward, "Unequal Error protection of H.264/AVCvideo Using Hierarchical QAM" Telekomunikacioni forum TELFOR 2008 Srbija, Beograd, novembar 25.-27., 2008.

[4] V.Theodorakopoulos,M.E.Woodward and K.Sotiropoulou "A dual priority M-QAM transmission system for high quality video over Mobile channels", in Proc. Of IEEE DFMA”05,pp.218-225,Feb 2005.

[5] Hamid Gharavi "Pilot- Assisted 16-level QAM for wireless video",IEEE trans on CIRC SYST VID, Vol.12, No 2, pp. 77-89, Feb. 2002.

[6] R .Stedman, H. Gharavi, L. Hanzo, and R.Steel, IEEE trans. Circuit Syst. Video Technol, vol. 6,pp 15-26 feb.1993.

[7] Radim ŠTUKAVEC, Tomáš KRATOCHVÍL, "Simulation and Measurement of the Transmission Distortions of the Digital Television DVB-T/H" RADIOENGINEERING, VOL. 19, NO. 3, SEPTEMBER 2010

[8] Farid Ghani, Md. Abdul Kader, and R. Badlishah Ahmed "Development and performance evaluation of HQAM for image transmission over wireless channels" IEEE 2011.

[9] Potential benefits of Hierarchical modes of DVB-T specification, Chris Nokes, Justin Mitchell.

[10] M. Mahdi Ghandi and M. Ghanbari, "Layered H.264 video transmission with hierarchical QAM," Elsevier J. Visual Communication Image Representation, Special issue on H.264/AVC, vol. 17, no. 2, pp. 451 - 466, April 2006.

[11] B. Barmada, E.V. Jones, Adaptive mapping and priority assignment for OFDM, in: Proceedings of the IEEE Conference of 3G Mobile Communication Technologies, London, 2002.

[12] K. K. V. Toh, H. Ibrahim, and M. N. Mahyuddin, "Saltand-pepper noise detection and reduction using fuzzy switching median filter," IEEE Trans. Consumer Electron., vol. 54, no. 4, pp. 1956-1961, Nov. 2008. 